\title{
Heavy-quark meson spectrum tests of the Oktay-Kronfeld action
}

\author{
Jon A. Bailey ${ }^{1}$, Carleton DeTar ${ }^{2, a}$, Yong-Chull Jang ${ }^{1,6, b}$, Andreas S. Kronfeld ${ }^{3,4, c}$, Weonjong Lee ${ }^{1, d}$, \\ Mehmet B. Oktay ${ }^{2,5}$ \\ ${ }^{1}$ Department of Physics and Astronomy, Lattice Gauge Theory Research Center, FPRD, and CTP, Seoul National University, Seoul 151-747, \\ South Korea \\ 2 Department of Physics and Astronomy, University of Utah, Salt Lake City, UT 84112, USA \\ 3 Theoretical Physics Department, Fermi National Accelerator Laboratory, Batavia, IL 60510, USA \\ ${ }^{4}$ Institute for Advanced Study, Technische Universität München, 85748 Garching, Germany \\ ${ }^{5}$ Department of Physics and Astronomy, University of Iowa, Iowa City, IA 52242, USA \\ ${ }^{6}$ Present address: Theoretical Division T-2, Los Alamos National Laboratory, Los Alamos, NM 87545, USA
}

Received: 9 May 2017 / Accepted: 28 September 2017 / Published online: 15 November 2017

(C) The Author(s) 2017. This article is an open access publication

\begin{abstract}
The Oktay-Kronfeld (OK) action extends the Fermilab improvement program for massive Wilson fermions to higher order in suitable power-counting schemes. It includes dimension-six and -seven operators necessary for matching to QCD through order $\mathrm{O}\left(\Lambda_{\mathrm{QCD}}^{3} / m_{Q}^{3}\right)$ in $\mathrm{HQET}$ power counting, for applications to heavy-light systems, and $\mathrm{O}\left(v^{6}\right)$ in NRQCD power counting, for applications to quarkonia. In the Symanzik power counting of lattice gauge theory near the continuum limit, the $\mathrm{OK}$ action includes all $\mathrm{O}\left(a^{2}\right)$ and some $\mathrm{O}\left(a^{3}\right)$ terms. To assess whether the theoretical improvement is realized in practice, we study combinations of heavy-strange and quarkonia masses and mass splittings, designed to isolate heavy-quark discretization effects. We find that, with one exception, the results obtained with the tree-level-matched OK action are significantly closer to the continuum limit than those obtained with the Fermilab action. The exception is the hyperfine splitting of the bottomstrange system, for which our statistical errors are too large to draw a firm conclusion. These studies are carried out with data generated with the tadpole-improved Fermilab and OK actions on 500 gauge configurations from one of MILC's $a \approx$ $0.12 \mathrm{fm}, N_{f}=2+1$-flavor, asqtad-staggered ensembles.
\end{abstract}

\section{Introduction}

Lattice QCD has been used to calculate nonperturbatively hadronic matrix elements needed for many aspects of Stan-

\footnotetext{
a e-mail: detar@physics.utah.edu

be-mail: ypj@lanl.gov

ce-mail: ask@fnal.gov

de-mail:wlee@snu.ac.kr
}

dard Model (SM) phenomenology [1,2]. Simulating heavy quarks in lattice QCD is especially challenging [3], because the lattice spacing may be of similar size to, or even larger than, the quark Compton wavelength. In this paper we examine a way to control discretization effects for massive (i.e., $m_{Q} \gg \Lambda_{\mathrm{QCD}}$ ) fermions in lattice gauge theory, building on the Fermilab action [4]. The key is to note that the dynamics of a quark confined by QCD takes place at shorter distances: of order $m v$ and $m v^{2}$ in quarkonia [5-9] and of order $\Lambda_{\mathrm{QCD}}$ in heavy-light systems [10-13]. This physics permits an understanding of heavy-quark discretization effects via heavy-quark effective theory (HQET) [14-16] or nonrelativistic QCD (NRQCD) [17].

These tools have been used to extend the Fermilab action [4] to an improvement (in the Symanzik [18] sense) known as the Oktay-Kronfeld (OK) action [19]. Whereas NRQCD [5-9] and HQET [10-13] are nonrelativistic effective field theories treating heavy quarks as two-component spinors, these actions are based on the Wilson clover action $[20,21]$ with four-component spinors. To retain a suitable remnant of Lorentz symmetry in physical quantities, the lattice action must have a time-space axis-interchange asymmetric form [4]. The action is then tuned with explicitly (bare) mass dependent couplings $c_{i}\left(m_{0} a\right)$ to connect smoothly the large $m_{Q} a>1$ and small $m_{Q} a \ll 1$ mass limits. In this way, the discretization errors can be controlled for arbitrary quark mass. In addition, a continuum limit without fine tuning is possible with the Fermilab action, which is not the case with direct discretizations of NRQCD.

The OK action [19] incorporates all dimension-six and certain dimension-seven bilinear operators. Although there are many such operators, many can be eliminated as redundant. Furthermore, only six are needed for tree-level match- 
ing to QCD through $\mathrm{O}\left(\lambda^{3}\right)\left(\lambda \sim \Lambda_{\mathrm{QCD}} / m_{Q}\right.$ or $\left.\Lambda_{\mathrm{QCD}} a\right)$ in HQET power counting for heavy-light mesons and $\mathrm{O}\left(v^{6}\right)$ ( $v$ being the relative velocity of the quark and antiquark) in NRQCD power counting for quarkonium. When $m_{0} a \ll$ 1, OK improvement reduces to $\mathrm{O}\left(a^{2}\right)$ with some $\mathrm{O}\left(a^{3}\right)$ terms in Symanzik [18] power counting. It is expected that the bottom- and charm-quark discretization errors could be reduced below the $1 \%$ level with the OK action [19].

The aim of this paper is to carry out two critical numerical tests to see whether the theoretical improvement [19] is achieved in practice. As we show below, the results are encouraging enough to proceed with related work: improving electroweak currents [22,23] and computing renormalization factors. The tests examine combinations of heavylight meson and quarkonium masses, designed to isolate discretization errors. For spin-independent improvements, we compute a combination of spin-averaged heavy-light and quarkonium masses discussed in Refs. [24,25]. For spindependent improvements, we compute the hyperfine splittings in each system.

The veracity of the spin-independent test is based on a nonrelativistic description of binding energies via the Breit equation $[25,26]$. This analysis homes in on errors in hadronic rest and kinetic masses. A by-product is an interesting wrinkle on the Fermilab "nonrelativistic interpretation," which forgoes the tuning of the rest mass. Other work with (essentially) the Fermilab action requires that the rest mass equal the kinetic mass $[27,28]$. Our analysis reveals that this step propagates errors in the kinetic binding energy to the rest mass, a point that may underappreciated; see Sect. 5.

The lattice data for this work have been generated from a MILC asqtad-staggered $N_{f}=2+1$ ensemble with $a \approx 0.12 \mathrm{fm}$ [29], which is coarse enough to possess clear heavy-quark discretization effects with the Fermilab action. We use an optimized conjugate-gradient inverter [30] to calculate quark propagators with both the tadpole-improved Fermilab and the OK actions over ranges spanning the $b$ and $c$ quarks. For the $b$-like and $c$-like quarks, the data are generated with four different values of the hopping parameter for the OK action, and two for the Fermilab action. Tadpole improvement of all terms is fully implemented in the conjugate-gradient program, as in an early, preliminary study [31]. Preliminary reports of the analysis reported here can be found in Refs. [32,33].

The outline of this paper is as follows. In Sect. 2, we briefly review the $\mathrm{OK}$ action and discuss its tadpole improvement. In Sect. 3, we describe the correlators we generate and the fits we use to extract the lightest meson energies in each channel at each momentum. In Sect. 4, we describe the fits needed to extract the rest and kinetic masses of the pseudoscalar and vector mesons. In Sect. 5, we assess the improvement from higher-order kinetic terms in the $\mathrm{OK}$ action with the mass combination of Refs. [24,25]. In Sect. 6, we assess the improvement from higher-order chromomagnetic interactions in the OK action by inspecting the difference of the hyperfine splittings of the rest and kinetic masses. We conclude in Sect. 7.

\section{Oktay-Kronfeld action}

The Oktay-Kronfeld (OK) action [19] $S_{\mathrm{OK}}$ is an improved version of the Fermilab action [4] $S_{\mathrm{FL}}$, which takes the clover action [21] for Wilson fermions [20], but chooses time-space asymmetric couplings:

$$
\begin{aligned}
S_{\mathrm{FL}} & =S_{0}+S_{B}+S_{E}, \\
S_{\mathrm{OK}} & =S_{\mathrm{FL}}+S_{\text {new }} .
\end{aligned}
$$

The explicit form of each piece $S_{0}, S_{B}, S_{E}$, and $S_{\text {new }}$, including many redundant operators and several operators not needed for tree-level matching, can be found in Ref. [19]. Here, we give an appropriate form for numerical simulations with tree-level tadpole improvement [34]. We write the action with gauge covariant translation operators $T_{ \pm \mu} \psi(x)=$ $U_{ \pm \mu}(x) \psi(x \pm a \hat{\mu})$ with $U_{-\mu}(x)=U_{\mu}^{\dagger}(x-a \hat{\mu})$.

We write the action with dimensionless fields $E_{x, i}, B_{x, i}$, $\psi_{x}$, and $\bar{\psi}_{x}$,

$E_{x, i}=a^{2} E_{i}(x)$,

$B_{x, i}=a^{2} B_{i}(x)$,

$\psi_{x}=a^{3 / 2} \psi(x)$,

$\bar{\psi}_{x}=a^{3 / 2} \bar{\psi}(x)$,

where the chromomagnetic field $B_{i}(x)$ and chromoelectric field $E_{i}(x)$ are

$$
\begin{aligned}
& B_{i}(x)=\frac{1}{2} \sum_{j, k=1}^{3} \varepsilon_{i j k} F_{j k}(x), \\
& E_{i}(x)=F_{4 i}(x),
\end{aligned}
$$

and

$$
F_{\mu \nu}=\frac{1}{8 a^{2}} \sum_{\rho= \pm \mu} \sum_{\sigma= \pm \nu} \operatorname{sgn}(\rho \sigma)\left[T_{\rho} T_{\sigma} T_{-\rho} T_{-\sigma}-\text { H.c. }\right]
$$

is the four-leaf-clover field strength.

Once the action is written in terms of the translation operators $T_{\mu}$, tadpole improvement can be carried out with the following steps [4]: Rewrite the gauge covariant translation operators $T_{ \pm \mu}$ in terms of tadpole-improved ones $\tilde{T}_{ \pm \mu}$ by using $T_{ \pm \mu}=u_{0} \tilde{T}_{ \pm \mu}$; replace the couplings $c_{i} \rightarrow \tilde{c}_{i}$, $r_{s} \rightarrow \tilde{r}_{s}, \zeta \rightarrow \tilde{\zeta}$, and $m_{0} \rightarrow \tilde{m}_{0}$ (the $u_{0}$ factors are absorbed into the couplings $\tilde{c}_{i}, \tilde{r}_{s}, \tilde{\zeta}$, and $\tilde{m}_{0}$ ); and finally, multiply each term of the action by the factor $u_{0}$ to recover the original form of the action. Then we arrive at the tadpole-improved OK action in terms of the unimproved translation operators, 
explicit tadpole factors, the tadpole-improved couplings $\tilde{c}_{i}$, and the hopping parameter $\kappa$ :

$$
\begin{aligned}
& \kappa=\tilde{\kappa} / u_{0}, \\
& \frac{1}{2 \tilde{\kappa}}=\tilde{m}_{0} a+\left(1+3 \tilde{r}_{s} \tilde{\zeta}+18 \tilde{c}_{4}\right) .
\end{aligned}
$$

The OK action then is

$$
\begin{aligned}
& S_{\mathrm{OK}}=\frac{1}{2 \kappa} \bar{\psi}_{x} \psi_{x} \\
& -\frac{1}{2} \bar{\psi}_{x}\left(1-\gamma_{4}\right) T_{4} \psi_{x}-\frac{1}{2} \bar{\psi}_{x}\left(1+\gamma_{4}\right) T_{-4} \psi_{x} \\
& -\frac{1}{2} \bar{\psi}_{x} \sum_{i=1}^{3}\left(\tilde{r}_{s} \tilde{\zeta}+8 \tilde{c}_{4}-\gamma_{i}\left(\tilde{\zeta}-2 \tilde{c}_{1}-12 \tilde{c}_{2}\right)\right) T_{i} \psi_{x} \\
& -\frac{1}{2} \bar{\psi}_{x} \sum_{i=1}^{3}\left(\tilde{r}_{s} \tilde{\zeta}+8 \tilde{c}_{4}+\gamma_{i}\left(\tilde{\zeta}-2 \tilde{c}_{1}-12 \tilde{c}_{2}\right)\right) T_{-i} \psi_{x} \\
& +\frac{\left(\tilde{c}_{1}+2 \tilde{c}_{2}\right)}{2 u_{0}} \bar{\psi}_{x} \sum_{i=1}^{3} \gamma_{i}\left(T_{i} T_{i}-T_{-i} T_{-i}\right) \psi_{x} \\
& +\frac{\tilde{c}_{4}}{u_{0}} \bar{\psi}_{x} \sum_{i=1}^{3}\left(T_{i} T_{i}+T_{-i} T_{-i}\right) \psi_{x} \\
& +\frac{\tilde{c}_{2}}{2 u_{0}} \bar{\psi}_{x} \sum_{i=1}^{3} \sum_{j \neq i}^{3} \gamma_{i}\left\{\left(T_{i}-T_{-i}\right),\left(T_{j}+T_{-j}\right)\right\} \psi_{x} \\
& +i \frac{\tilde{c}_{5}}{4 u_{0}^{2}} \bar{\psi}_{x} \sum_{i=1}^{3} \Sigma_{i} T_{i}^{(3)} \psi_{x}-\frac{\tilde{c}_{E} \tilde{\zeta}}{2 u_{0}^{3}} \bar{\psi}_{x} \sum_{i=1}^{3} \alpha_{i} E_{x, i} \psi_{x} \\
& -i \frac{\left(\tilde{c}_{B} \tilde{\zeta}+16 \tilde{c}_{5}\right)}{2 u_{0}^{3}} \bar{\psi}_{x} \sum_{i=1}^{3} \Sigma_{i} B_{x, i} \psi_{x} \\
& +\frac{\tilde{c}_{E E}}{2 u_{0}^{4}} \bar{\psi}_{x} \sum_{i=1}^{3} \gamma_{i}\left[\left(T_{4}-T_{-4}\right), E_{x, i}\right] \psi_{x} \\
& +i \frac{\tilde{c}_{3}}{2 u_{0}^{4}} \bar{\psi}_{x} \sum_{i=1}^{3} \gamma_{i} \Sigma_{i}\left\{\left(T_{i}-T_{-i}\right), B_{x, i}\right\} \psi_{x} \\
& +i \frac{\tilde{c}_{3}}{2 u_{0}^{4}} \bar{\psi}_{x} \sum_{i=1}^{3} \sum_{j \neq i}^{3} \gamma_{i} \Sigma_{j}\left[\left(T_{i}-T_{-i}\right), B_{x, j}\right] \psi_{x} \\
& +i \frac{\tilde{c}_{5}}{u_{0}^{4}} \bar{\psi}_{x} \sum_{i=1}^{3}\left(-\frac{1}{4} \Sigma_{i} T_{i}^{(3)}\right. \\
& \left.+\sum_{j \neq i}^{3}\left\{\Sigma_{i} B_{x, i},\left(T_{j}+T_{-j}\right)\right\}\right) \psi_{x},
\end{aligned}
$$

where

$$
T_{i}^{(3)} \equiv \sum_{j, k=1}^{3} \varepsilon_{i j k}\left[T_{-k}\left(T_{j}-T_{-j}\right) T_{k}-T_{k}\left(T_{j}-T_{-j}\right) T_{-k}\right]
$$

is the three-link part of the $c_{5}$ term (which is composed of three- and five-link terms), $i=1,2,3$ denote the spatial directions, and a sum over sites $x \in \mathbb{Z}^{4}$ is implied. The matrices $\alpha_{i}$ and $\Sigma_{i}$ are defined by

$$
\begin{aligned}
\sigma^{\mu \nu} & =\frac{i}{2}\left[\gamma^{\mu}, \gamma^{\nu}\right], \\
\sigma^{4 i} & =i \sigma^{0 i}=i \alpha^{i}, \\
\sigma^{i j} & =-\varepsilon^{i j k} \Sigma_{k},
\end{aligned}
$$

where $\gamma^{\mu}$ are the Dirac matrices, and the totally antisymmetric tensor component $\varepsilon^{123}=1$. We have coded this form of the tadpole-improved OK action with the USQCD [35] software QOPQDP and the MILC code, with the optimization scheme discussed in Ref. [30].

The tadpole-improved couplings $\tilde{c}_{i}$ are obtained by applying the matching conditions in Ref. [19], substituting $\tilde{m}_{0} a$ for $m_{0} a, \tilde{r}_{s}$ for $r_{s}, \tilde{\zeta}$ for $\zeta$, and using Eq. (11); in practice, $\tilde{r}_{s}=r_{s}$ and $\tilde{\zeta}=\zeta$. The redundant coefficient of the Wilson term, $r_{s}$, is set to one to lift the unwanted fermion doublers as usual. The tuning parameter $\zeta=\kappa_{s} / \kappa_{t}$ plays the following role. Recall the definitions of the rest mass $m_{1}$ and the kinetic mass $m_{2}$, from the energy $E(\boldsymbol{p})$ :

$$
\begin{aligned}
& m_{1}=E(\mathbf{0}), \\
& \frac{1}{m_{2}}=\left.\frac{\partial^{2} E}{\partial p_{i}^{2}}\right|_{p=\mathbf{0}} .
\end{aligned}
$$

At the tree level $[4,19]$,

$$
\begin{aligned}
& m_{1} a=\ln \left(1+m_{0} a\right), \\
& \frac{1}{m_{2} a}=\frac{r_{s} \zeta}{1+m_{0} a}+\frac{2 \zeta^{2}}{m_{0} a\left(2+m_{0} a\right)},
\end{aligned}
$$

for both the Fermilab and the OK actions. Substituting $\tilde{m}_{0} a$ from Eq. (11) for $m_{0} a$ in Eqs. (19) and (20) yields tadpoleimproved tree-level masses, denoted $\tilde{m}_{1}$ and $\tilde{m}_{2}$. One can arrange for $m_{2}=m_{1}$ (or $\tilde{m}_{1}=\tilde{m}_{2}$ ) by tuning $\zeta$. In fact, this tuning can be carried out nonperturbatively with hadron masses - denoted in this paper $M_{1}$ and $M_{2}$. Unfortunately, in that case the discretization errors of $M_{2}$ are passed on to $M_{1}$.

In this paper, we set $\zeta=1$, which is just the Fermilab nonrelativistic interpretation [4], in which $m_{2}$ is taken as the physically relevant mass. In the splittings of hadron rest masses, the quark contribution $m_{1}$ cancels; the combinations of masses of the OK action presented in Sects. 5 and 6 are based on splittings and, hence, are immune to this choice. (The quark rest mass also does not affect matrix elements of local operators [14].) The couplings of the OK action in Eq. (12) $-\tilde{c}_{B}, \tilde{c}_{E}, \tilde{c}_{1}, \tilde{c}_{2}, \tilde{c}_{3}, \tilde{c}_{4}, \tilde{c}_{5}$, and $\tilde{c}_{E E}-$ are obtained by substituting $\tilde{m}_{0} a$ for $m_{0} a$ on the right-hand sides of Eqs. (4.72)-(4.79) in Ref. [19], with $\zeta=r_{s}=1$ [and $r_{E}=r_{E E}=0$ in Eqs. (4.78) and (4.79) of Ref. [19]].

Note that the rest mass $m_{1}$ and kinetic mass $m_{2}$ have the bare-mass dependences in Eqs. (19) and (20) for both the OK 
Table 1 Input hopping parameters $\kappa_{\mathrm{OK}}$ for the $\mathrm{OK}$ action and $\kappa_{\mathrm{FL}}$ for the Fermilab action. Note that these inputs are exact and are not chosen by tuning with a physical observable. The resulting kinetic meson masses cover the region of physical heavy-light and quarkonium masses at this lattice spacing, falling in two regions, referred to as $b$-like and $c$ like, respectively. Vertically aligned values yield approximately equal heavy-light meson masses

\begin{tabular}{llllllll}
\hline$Q$ & $b$-like & \multicolumn{7}{c}{$c$} & $c$-like \\
\hline$\kappa_{\mathrm{OK}}$ & 0.039 & 0.040 & 0.041 & 0.042 & 0.0468 & 0.048 & 0.049 \\
$\kappa_{\mathrm{FL}}$ & & & 0.083 & 0.091 & & 0.121 & 0.050 \\
\hline
\end{tabular}

and the Fermilab actions. However, the hopping parameter $\kappa$, Eq. (11), differs by the $c_{4}$ term in Eq. (12), which, along with the $c_{1}$ and $c_{2}$ terms, improves the $\mathrm{O}\left((a p)^{4}\right)$ terms in the dispersion relation [19].

\section{Meson correlators}

\subsection{Data description}

We use the MILC asqtad-staggered $N_{f}=2+1$ gauge ensemble that has dimensions $N_{L}^{3} \times N_{T}=20^{3} \times 64, \beta=6.79$, tree-level tadpole factor $u_{0}=0.8688$ from the plaquette, and lattice spacing $a \approx 0.12 \mathrm{fm}$ [29]. The asqtad-staggered action [36-40] is used for the light degenerate sea quarks with mass $a m_{l}=0.02$ and strange sea quark with mass $a m_{s}=0.05$. For the tests reported here, we use $N_{\mathrm{cfg}}=500$ of the approximately 2000 available configurations.

On each configuration, we use $N_{\text {src }}=6$ sources $\left(t_{i}, \boldsymbol{x}_{i}\right)$ for calculating valence quark propagators. The source time slices $t_{i}$ are evenly spaced along the lattice with a randomized offset $t_{0} \in[0,20)$ for each configuration. The spatial source coordinates $\boldsymbol{x}_{i}$ are randomly chosen within the spatial cube.

We compute two-point correlators

$C^{M}(t, \boldsymbol{p})=\sum_{\boldsymbol{x}} e^{\mathrm{i} \boldsymbol{p} \cdot \boldsymbol{x}}\left\langle\mathscr{O}^{M^{\dagger}}(t, \boldsymbol{x}) \mathscr{O}^{M}(0, \mathbf{0})\right\rangle$,

for heavy-strange mesons $M=\bar{Q} s$ and quarkonia $M=\bar{Q} Q$ with momentum $\boldsymbol{p}$ and heavy quarks $Q$ in the $b$ - and $c$-like regions. The interpolating operators $\mathscr{O}^{M}(x)$ are

$\mathscr{O}_{\mathrm{t}}^{\bar{Q} q}(x)=\bar{\psi}_{\alpha}(x) \Gamma_{\alpha \beta} \Omega_{\beta \mathrm{t}}(x) \chi(x)$,

${ }_{\mathscr{O}} \bar{Q} Q(x)=\bar{\psi}_{\alpha}(x) \Gamma_{\alpha \beta} \psi_{\beta}(x)$,

for heavy-light mesons and quarkonium, respectively. Here the heavy-quark field $\psi$ is the Wilson-type fermion appearing in the $\mathrm{OK}$ or Fermilab action, while the light-quark field $\chi$ is the staggered fermion appearing in the asqtadstaggered action. The taste degree of freedom for the staggered fermion is obtained from the one-component field $\chi$ with $\Omega(x)=\gamma_{1}^{x_{1}} \gamma_{2}^{x_{2}} \gamma_{3}^{x_{3}} \gamma_{4}^{x_{4}}[26,41]$. We generate pseudoscalar and vector-meson correlators with the spin structures $\Gamma=\gamma_{5}$ and $\gamma_{i}$ (for $i=1,2,3$ ), respectively.
Table 2 Values for the couplings in the OK action, computed from the input hopping parameters in Table 1, the critical hopping parameter $\kappa_{\text {crit }}=0.05354$, and the tadpole factor $u_{0}=0.8688$. The errors on $\kappa_{\text {crit }}$ and $u_{0}$ are unimportant for this study and, thus, neglected. Our code evaluates the improvement coefficients directly from the tree-level matching result as described in the text. Here, we present truncated values. The OK couplings $\tilde{c}_{B}=r_{s}=1, \tilde{c}_{4}=9 / 24$, and $\tilde{c}_{5}=1 / 4 \mathrm{do}$ not depend on the quark mass $\tilde{m}_{0} a$

\begin{tabular}{llllll}
\hline$\kappa$ & $\tilde{m}_{0} a$ & $\tilde{c}_{E}$ & $\tilde{c}_{1}$ & $\tilde{c}_{2}=\tilde{c}_{3}$ & $\tilde{c}_{E E}$ \\
\hline 0.039 & 4.0066 & 0.4398 & 0.6345 & -0.0706 & -0.0040 \\
0.040 & 3.6377 & 0.4540 & 0.5703 & -0.0733 & -0.0045 \\
0.041 & 3.2867 & 0.4697 & 0.5089 & -0.0763 & -0.0050 \\
0.042 & 2.9525 & 0.4870 & 0.4499 & -0.0795 & -0.0055 \\
0.0468 & 1.5471 & 0.6041 & 0.1924 & -0.0991 & -0.0095 \\
0.048 & 1.2397 & 0.6466 & 0.1322 & -0.1053 & -0.0108 \\
0.049 & 0.9950 & 0.6884 & 0.0823 & -0.1108 & -0.0119 \\
0.050 & 0.7601 & 0.7374 & 0.0320 & -0.1164 & -0.0126 \\
\hline
\end{tabular}

We generate data with ten meson momenta $a \boldsymbol{p}=$ $2 \pi \boldsymbol{n} / N_{L}: \boldsymbol{n}=(0,0,0),(1,0,0),(1,1,0),(1,1,1),(2,0,0)$, $(2,1,0),(2,1,1),(2,2,0),(2,2,1),(3,0,0)$, including all permutations of the components. These momenta all satisfy $(\text { a p })^{2}<0.9$.

The hopping parameter values chosen for this study span the $b$ - and $c$-like regions for $a \approx 0.12 \mathrm{fm}$ and are given in Table 1. We fix the valence light-quark mass for the heavylight meson correlators to the strange sea quark mass $a m_{s}$. In anticipation of tuning runs for the OK action, we generate heavy-light and quarkonium correlators by using the $\mathrm{OK}$ action with four different values for the hopping parameter $\kappa_{\mathrm{OK}}$. For purposes of comparison, we simulate the $b$ - and $c$-like regions with two values each for the hopping parameter $\kappa_{\mathrm{FL}}$ of the Fermilab action. The numerical values of the OK couplings, which are obtained from the formulas in Ref. [19] with $\tilde{m}_{0} a=u_{0}\left[1 / 2 \kappa-1 / 2 \kappa_{\text {crit }}\right]$, are given to four digits in Table 2.

\subsection{Correlator fits}

The ground-state energies are extracted from correlator fits to the function 
Table 3 Bayesian priors used for two-point correlator fits. For heavylight mesons, the same priors are used for both heavy quarks $Q=c, b$. For bottomonium, we present a fit without the excited state, i.e., $R_{1}=0$ in Eq. (25)

\begin{tabular}{lllllll}
\hline Meson & $R_{1}$ & $\Delta E_{1}$ & $R_{0}^{p}$ & $\Delta E_{0}^{p}$ & $R_{1}^{p}$ & $\Delta E_{1}^{p}$ \\
\hline $\bar{Q} q$ & $3.0(1.5)$ & $0.5(3)$ & $0.2(2)$ & $0.1(2)$ & $3.0(1.5)$ & $0.5(4)$ \\
$\bar{c} c$ & $1.5(1.0)$ & $0.5(4)$ & & & & \\
\hline
\end{tabular}

$$
\begin{aligned}
f(t) & =g(t)+g(T-t), \\
g(t) & =\sum_{i=0,1}\left[A_{i} e^{-E_{i} t}-(-1)^{t} A_{i}^{p} e^{-E_{i}^{p} t}\right] \\
& =A_{0} e^{-E_{0} t} \sum_{i=0,1}\left[R_{i} e^{-\Delta E_{i} t}-(-1)^{t} R_{i}^{p} e^{-\Delta E_{i}^{p} t}\right],
\end{aligned}
$$

where $A_{0}$ is the ground-state amplitude, and $A_{1}$ is the first excited-state amplitude. We also incorporate the staggered parity partner state with amplitude $A_{i}^{p}$ and energy $E_{i}^{p}$ into the fit function. In practice, we take an amplitude ratio $R_{i}^{(p)}=$ $A_{i}^{(p)} / A_{0}$ and energy difference $\Delta E_{i}^{(p)}=E_{i}^{(p)}-E_{i-1}^{(p)}$ as fit parameters instead of $A_{i}^{(p)}$ and $E_{i}^{(p)}$. By definition $R_{0}=1$, $\Delta E_{0}=0, E_{-1}^{p} \equiv E_{0}$, and $R_{0}$ and $\Delta E_{0}$ are not fit parameters. The parity partners are not present for quarkonium, because they do not contain (valence) staggered fermions, so we set $R_{i}^{p}=0$ for quarkonium. The fits are carried out with the Bayesian priors given in Table 3.

We carry out correlated fits with a statistical estimate of the covariance matrix $\operatorname{cov}\left(t, t^{\prime}\right)$ between different time slices $t$ and $t^{\prime}$,

$\operatorname{cov}\left(t, t^{\prime}\right)=\frac{1}{\mathscr{N}} \sum_{i=1}^{N_{\mathrm{cfg}}}\left[C_{i}(t)-C(t)\right]\left[C_{i}\left(t^{\prime}\right)-C\left(t^{\prime}\right)\right]$,

where the normalization factor $\mathscr{N}=N_{\text {cfg }}\left(N_{\text {cfg }}-1\right), C_{i}(t)$ represents the average over the $N_{\text {src }}$ sources for the $i^{\text {th }}$ gauge field, and the two-point correlator $C(t)=C^{M}(t, p)$ for a given meson type $M$ and momentum $\boldsymbol{p}$ is estimated with the ensemble average

$C(t)=\frac{1}{N_{\mathrm{cfg}}} \sum_{i=1}^{N_{\mathrm{cfg}}} C_{i}(t)$

To increase statistics, we take advantage of the structure of Eq. (24) and average the data for time separations $t$ and $T-t$. Then we take the fit interval $\left[t_{\min }, t_{\max }\right]$, where $0<t_{\min }<t_{\max }<T / 2$, equal to [7, 15] for the heavylight meson with the OK action and $[9,15]$ with the Fermilab action. For charmonium, the fit interval is $[9,14]$ for both actions. For bottomonium, the fit interval is [12, 17] for the OK action and [14, 19] for the Fermilab action. We fix these intervals for fits to all correlators, independent of hop-

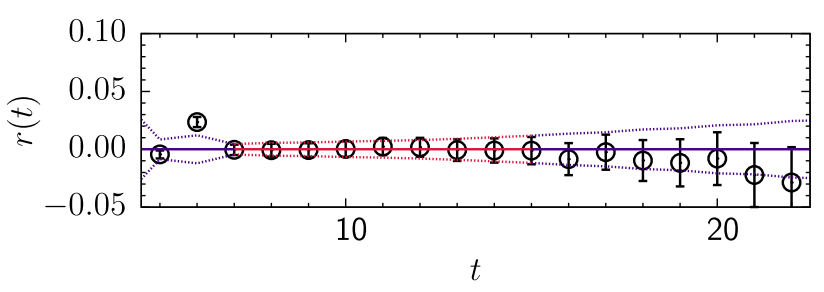

(a) Residual

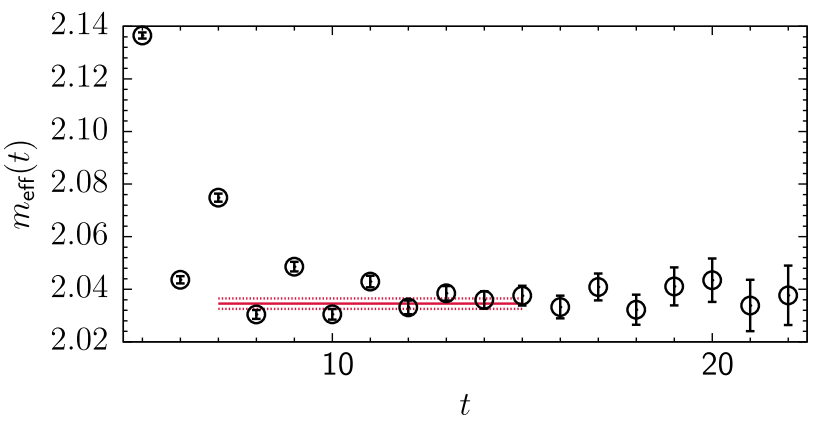

(b) Effective Mass

Fig. 1 Correlated fit results for a pseudoscalar heavy-light meson correlator generated with the $\mathrm{OK}$ action at $\kappa=0.041$ and $\boldsymbol{p}=\mathbf{0}$. a Residual defined in Eq. (29) and its statistical error from a fit over $7 \leq t \leq 15$; the kinks for $t<7$ are a remnant of the oscillating, wrong-parity states, seen in the right-hand side of Eq. (25). b Effective mass (points) defined in Eq. (28) with fit result for the ground-state energy $E$ (horizontal red line plotted over the chosen fit interval)

ping parameter $\kappa$, momentum $\boldsymbol{p}$, and pseudoscalar vs. vector channel.

The $t_{\min }$ are chosen by observing the effective mass, defined by

$m_{\mathrm{eff}}(t)=\frac{1}{2} \ln \left(\frac{C^{M}(t)}{C^{M}(t+2)}\right)$

as well as comparing the fit results $E$ with the $m_{\mathrm{eff}}(t)$. Figure 1 shows the effective mass $m_{\mathrm{eff}}(t)$ and correlator fit residual $r(t)$

$r(t)=\frac{C^{M}(t)-f(t)}{\left|C^{M}(t)\right|}$

for a pseudoscalar heavy-light meson correlator generated with the $\mathrm{OK}$ action, $\kappa_{\mathrm{OK}}=0.041$, and $\boldsymbol{p}=\mathbf{0}$. The pseudoscalar bottomonium correlator fit results obtained with the same action and parameters are given in Fig. 2. To estimate the statistical errors, we use a single-elimination jackknife.

A feature of our $b$-like heavy-light correlators is that they are somewhat more precise for the $\mathrm{OK}$ action than for the Fermilab action. At the same time $b$-like quarkonium and both types of $c$-like correlators are similarly precise for the two actions. We do not understand the reason for this behavior. Of course, it propagates to the meson masses and their combinations examined in the next sections. 


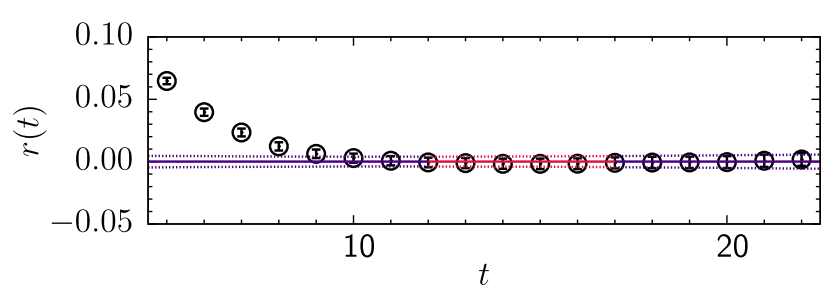

(a) Residual

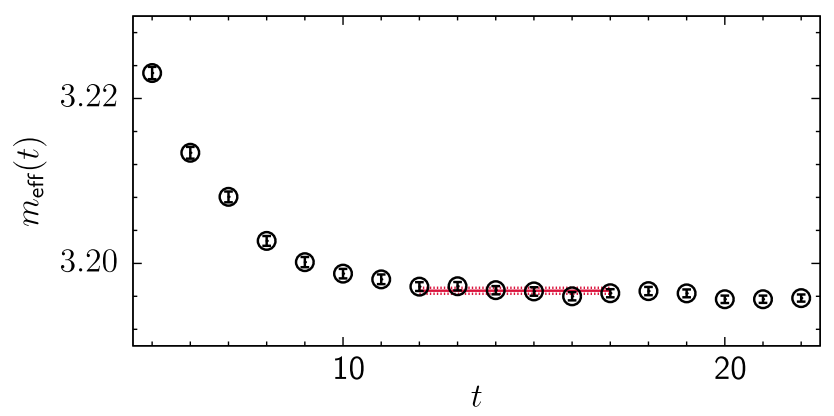

(b) Effective Mass

Fig. 2 Correlated fit results for a pseudoscalar bottomonium correlator generated with the $\mathrm{OK}$ action at $\kappa=0.041$ and $\boldsymbol{p}=\mathbf{0}$. a Residual defined in Eq. (29) and its statistical error from a fit over $12 \leq t \leq 17$. b Effective mass (points) defined in Eq. (28) with fit result for the ground-state energy $E$ (horizontal red line plotted over the chosen fit interval)

\section{Meson masses}

The dispersion relation of the mesons has the same form as that of the quarks with the quark masses $m_{1}$ and $m_{2}$ given in Eqs. (17) and (18), respectively, replaced by meson masses $M_{1}$ and $M_{2}$. The mismatch between the meson rest and kinetic masses can be exploited to test the improvement of nonrelativistically interpreted actions.

We fit the ground-state energy $E(\boldsymbol{p})$ in Eq. (25) for each momentum $\boldsymbol{p}$ to the nonrelativistic dispersion relation to obtain the rest mass $M_{1}$ and kinetic mass $M_{2}$ of each meson. Including terms up to $\mathrm{O}\left(\boldsymbol{p}^{6}\right)$, the dispersion relation is

$$
\begin{aligned}
E(\boldsymbol{p}) & =M_{1}+\frac{\boldsymbol{p}^{2}}{2 M_{2}}-\frac{\left(\boldsymbol{p}^{2}\right)^{2}}{8 M_{4}^{3}}+E_{4}^{\prime}+E_{6}+E_{6}^{\prime}, \\
E_{4}^{\prime} & =-\frac{a^{3} W_{4}}{6} \sum_{i} p_{i}^{4}, \\
E_{6} & =\frac{\left(\boldsymbol{p}^{2}\right)^{3}}{16 M_{6}^{5}}, \\
E_{6}^{\prime} & =\frac{a^{5} W_{6}^{\prime}}{2} \boldsymbol{p}^{2} \sum_{i} p_{i}^{4}-\frac{a^{5} W_{6}}{3} \sum_{i} p_{i}^{6} .
\end{aligned}
$$

The $M_{4,6}$ are generalized masses; the rest mass $M_{1}$ and these generalized masses $M_{4,6}$ are expected $[4,19]$ to approach the kinetic mass $M_{2}$ in the continuum limit. The $O(3)$ rotationsymmetry-breaking terms are $E_{4}^{\prime}$ and $E_{6}^{\prime}$. In the continuum limit, the coefficients $a^{3} W_{4}, a^{5} W_{6}^{\prime}$, and $a^{5} W_{6}$ vanish. We fit the simulation data for $E$ ( $\boldsymbol{p})$ to the right-hand side of Eq. (30), taking the full covariance matrix among the different momentum channels, and investigate variations by excluding some or all of the higher-order terms $E_{4}^{\prime}, E_{6}$, and $E_{6}^{\prime}$. We do not introduce priors here. The seven fit parameters are $M_{1}, M_{2}^{-1}$, $M_{4}^{-3}, M_{6}^{-5}, W_{4}, W_{6}^{\prime}$, and $W_{6}$. We find that we do not need all seven terms in the dispersion relation. In many cases, it suffices to keep only the first five, while some fits give better $p$ values with the first six.

For the pseudoscalar heavy-light meson, as the spectrum becomes more relativistic, $M_{2}$ approaches $M_{1}$, and including the $E_{6}$ term results in a better fit. Because the vector heavylight meson spectrum has a larger statistical error than the pseudoscalar-meson spectrum, the $E_{6}$ term is not only determined to be statistically zero, but also does not improve the fit. The $E_{6}$ term also improves the fit for the charmonium spectrum with the Fermilab action.

The $W_{6}^{\prime}$ term in addition to the $E_{6}$ term improves the fit for the bottomonium spectrum with the Fermilab action. Note that the correlator fit for bottomonium does not include any excited states, although including a single excited state is statistically consistent, when fitting Eq. (30) without the $E_{6}$ and $E_{6}^{\prime}$ terms. We use the fits with excited states to crosscheck the single-state fits. We observed the same behavior with the two-state fit for the bottomonium spectrum with the $\mathrm{OK}$ action. Note that the bottomonium spectrum for the $\mathrm{OK}$ action still results in a good fit without the $E_{6}$ and $E_{6}^{\prime}$ terms, when the correlator fit only accounts for the ground state.

Figure 3 shows the dispersion relation fits to the pseudoscalar heavy-light meson and quarkonium data generated with the OK (Fermilab) action with $\kappa_{\mathrm{OK}}=0.041$ $\left(\kappa_{\mathrm{FL}}=0.083\right)$. We plot the dispersion relation fit results after subtracting the rest mass and the higher-order term $E_{4}^{\prime}$. We thus define

$\widetilde{E}=E-M_{1}-E_{4}^{\prime}$

to draw the plot. As one can see from Fig. 3, the slope - and, hence, the kinetic mass $M_{2}$ - is reliably determined from fits to Eq. (30). Interestingly, the OK action leads to statistical errors noticeably smaller than those from the Fermilab action, especially for the heavy-light masses.

The fit results for the heavy-light mesons are summarized in Table 4a, b. They are obtained from correlated fits. From these tables for the heavy-light mesons, one can see that the higher-order generalized mass $M_{4}$ approaches the kinetic mass $M_{2}$ as the kinetic mass decreases. The rest mass $M_{1}$ also approaches the kinetic mass $M_{2}$. Note that $M_{4}$ does not necessarily agree better with $M_{2}$ for the OK action, even though the $\mathrm{OK}$ action tunes the action such that $m_{4}=m_{2}$. A possible explanation is that the binding energy $M_{4}-m_{4}$ stems 


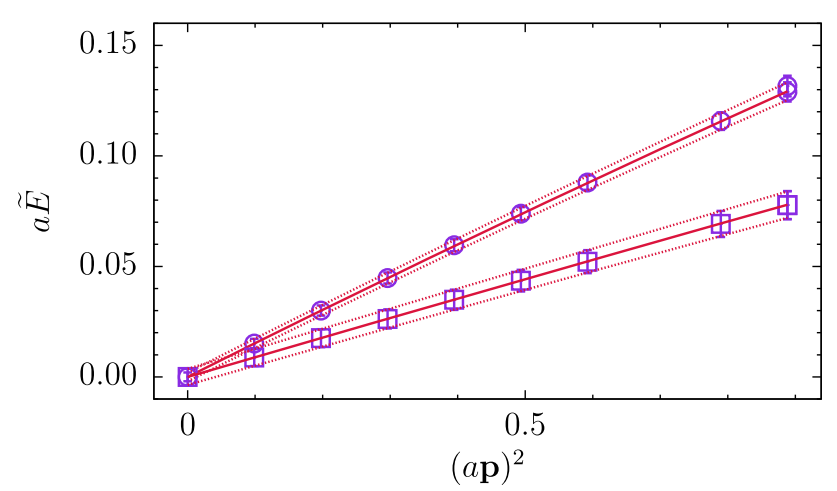

(a) $\mathrm{OK}$

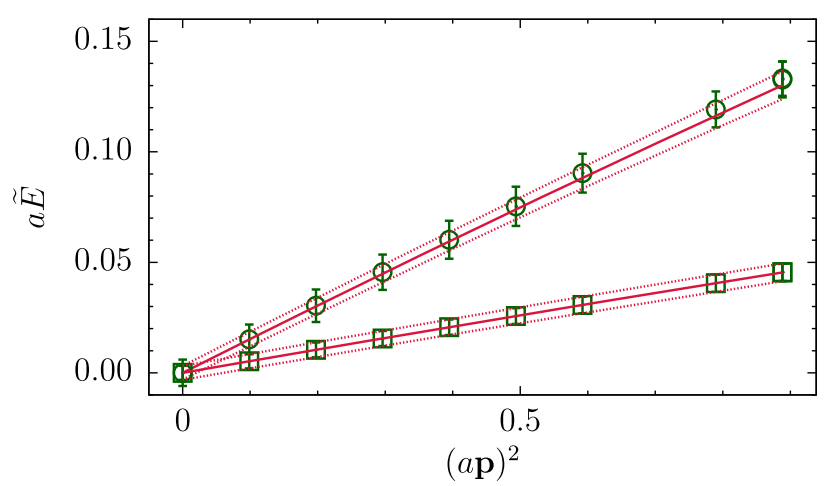

(b) Fermilab

Fig. 3 The pseudoscalar heavy-light meson (circle) and quarkonium (square) subtracted energies $\widetilde{E}$ [Eq. (34)] as a function of $(a \boldsymbol{p})^{2}$ for a the OK action at $\kappa=0.041$ and $\mathbf{b}$ the Fermilab (clover) action at $\kappa=0.083$. The lines show fits to Eq. (30). The errors are from a single-elimination jackknife. In these plots, the errors for the bottomonium data points and fit lines are scaled by a factor 10 . The behavior for vector mesons is similar, apart from the larger statistical errors

from higher-dimension effects not yet incorporated into the OK action.

The correlated fit results for quarkonia are summarized in Table 5a, b. For the charmonium results obtained with the OK action, $M_{4}$ is closer to the kinetic mass $M_{2}$ than to the rest mass $M_{1}$, but for the Fermilab action $M_{4}$ is closer to $M_{1}$. In the bottomonium region, $M_{4}$ is closer to the rest mass $M_{1}$ than the kinetic mass $M_{2}$ for both actions. The difference $M_{2}-$ $M_{1}$ is large for the Fermilab action at $\kappa_{\mathrm{FL}}=0.083,0.091$, but it is diminished with the $\mathrm{OK}$ action at the comparable values $\kappa_{\mathrm{OK}}=0.039,0.040,0.041,0.042$. However, even with the $\mathrm{OK}$ action, the difference between $M_{4}$ and $M_{2}$ in the bottomonium spectra is larger than that in the heavy-light meson spectra.

\section{The "inconsistency" [24]}

To study how the Fermilab method works, Ref. [24] introduced the quantity
$I=\frac{2 \delta M_{\bar{Q} q}-\left(\delta M_{\bar{Q} Q}+\delta M_{\bar{q} q}\right)}{2 M_{2 \bar{Q} q}}$

where

$\delta M_{\bar{Q} q}=M_{2} \bar{Q}_{q}-M_{1} \bar{Q}_{q}$.

The combination $I$ should vanish, even when the quark rest masses $m_{1}$ are mistuned. If $I$ does not vanish, it means that the action contains nontrivial discretization effects at higher order in the HQET or NRQCD power counting, so $I$ is often called the "inconsistency." In fact, as we reproduce below, $I \neq 0$ for the Fermilab action [24] (unless $m_{2} a \ll 1$ ), as one can see in Eq. (40).

The explanation is as follows [25]. The meson masses $M_{i}$ $(i=1,2)$ can be written as a sum of the quark masses $m_{i}$ and the binding energy:

$M_{i \bar{Q} q}=m_{i \bar{Q}}+m_{i q}+B_{i \bar{Q} q}$,

which defines $B_{i}$. Upon substituting Eq. (37) into Eqs. (36) and (35), the quark masses cancel out, and the inconsistency becomes a relation among the binding energies,

$I=\frac{2 \delta B_{\bar{Q} q}-\left(\delta B_{\bar{Q} Q}+\delta B_{\bar{q} q}\right)}{2 M_{2 \bar{Q} q}}$

where

$\delta B_{\bar{Q}} q=B_{2} \bar{Q}_{q}-B_{1} \bar{Q}_{q}$.

The quantities in Eqs. (36), (37), and (39) for heavy $(\bar{Q} Q)$ and light $(\bar{q} q)$ quarkonium are defined similarly. Because light quarks always have $m a \ll 1$, the $\mathrm{O}\left((m a)^{2}\right)$ distinction between rest and kinetic masses is negligible for them, so we omit $\delta M_{\bar{q} q}$ (or $\delta B_{\bar{q} q}$ ) when computing $I$. The rest-mass binding energy $B_{1}$ is sensitive to effects of $\mathrm{O}(\lambda)$ or $\mathrm{O}\left(v^{2}\right)$, while the kinetic-mass binding energy $B_{2}$ is sensitive to effects of $\mathrm{O}\left(\lambda^{3}\right)$ or $\mathrm{O}\left(v^{4}\right)$, with their larger relative discretization errors (for the clover and Fermilab actions). The discretization errors can be studied in the nonrelativistic limit via the Breit equation, yielding $[25,26]$

$$
\begin{aligned}
\delta B_{\bar{Q} q}= & \frac{5}{3} \frac{\left\langle\boldsymbol{p}^{2}\right\rangle}{2 \mu_{2}}\left[\mu_{2}\left(\frac{m_{2 \bar{Q}}^{2}}{m_{4 \bar{Q}}^{3}}+\frac{m_{2 q}^{2}}{m_{4 q}^{3}}\right)-1\right] \\
& +\frac{4}{3} a^{3} \frac{\left\langle\boldsymbol{p}^{2}\right\rangle}{2 \mu_{2}} \mu_{2}\left[w_{4 \bar{Q}} m_{2 \bar{Q}}^{2}+w_{4 q} m_{2 q}^{2}\right]+\mathrm{O}\left(\boldsymbol{p}^{4}\right)
\end{aligned}
$$

in the $S$ wave, where the reduced mass $\mu_{2}^{-1}=m_{2 \bar{Q}}^{-1}+m_{2 q}^{-1}$, and $m_{2}, m_{4}$, and $w_{4}$ are defined through the quark analog of Eq. (30). Here, $\boldsymbol{p}$ is the relative momentum of $\bar{Q}$ and $q$ in their center of mass. The OK action matches $m_{4}=m_{2}$ and 
Table 4 Dispersion fit results for heavy-light mesons with masses near those of the $D_{s}^{(*)}$ and $B_{s}^{(*)}$. The energies $E$ are from the correlator fits to the function in Eq. (30). The first column indicates the lattice action and the (approximate) heavy-quark flavor. The second column is the hopping parameter $\kappa$. The following columns are the rest mass $M_{1}$, the kinetic mass $M_{2}$, the generalized masses $M_{4}$ and $M_{6}$, and the coefficient of an $O(3)$ symmetry breaking term $W_{4}$. The last two columns are the $\chi^{2}$ divided by the degrees of freedom (dof) $N_{\text {data }}-N_{\text {param }}, p$-value. All ten momenta data points are included in the dispersion relation fit; these spectra are obtained from the correlator fits to Eq. (25) which include all correlations. Fits to the dispersion relation include all correlations among different momenta. Errors are all from a single-elimination jackknife

\begin{tabular}{|c|c|c|c|c|c|c|c|c|}
\hline Action $(Q)$ & $\kappa$ & $M_{1}$ & $M_{2}$ & $M_{4}$ & $W_{4} \times 10^{2}$ & $M_{6}$ & $\chi^{2} /$ dof & $p$ \\
\hline \multicolumn{9}{|c|}{ (a) Pseudoscalar } \\
\hline \multirow[t]{4}{*}{ OK (b-like) } & 0.039 & $2.2141(24)$ & $3.924(63)$ & $2.50(16)$ & $-6.1(7)$ & - & $1.13(4)$ & $0.34(2)$ \\
\hline & 0.040 & $2.1261(22)$ & $3.580(53)$ & $2.46(15)$ & $-5.5(8)$ & - & $1.21(4)$ & $0.30(2)$ \\
\hline & 0.041 & $2.0345(19)$ & $3.256(45)$ & $2.40(15)$ & $-4.9(9)$ & - & $1.26(4)$ & $0.27(2)$ \\
\hline & 0.042 & $1.9382(17)$ & $2.952(37)$ & $2.33(15)$ & $-4.0(1.0)$ & - & $1.35(4)$ & $0.23(2)$ \\
\hline \multirow[t]{4}{*}{ OK (c-like) } & 0.0468 & $1.3742(9)$ & $1.663(12)$ & $1.59(7)$ & $3.0(2.4)$ & - & $1.29(4)$ & $0.26(2)$ \\
\hline & 0.048 & $1.1861(8)$ & $1.362(8)$ & $1.33(4)$ & $6.8(3.5)$ & $1.4(2)$ & $1.47(5)$ & $0.20(2)$ \\
\hline & 0.049 & $1.0003(7)$ & $1.104(6)$ & $1.12(3)$ & $7.3(5.5)$ & $1.2(1)$ & $1.47(5)$ & $0.20(2)$ \\
\hline & 0.050 & $0.7688(5)$ & $0.827(5)$ & $0.85(2)$ & $7.4(10.2)$ & $1.0(1)$ & $0.32(2)$ & $0.90(1)$ \\
\hline \multirow[t]{2}{*}{ FL (b-like) } & 0.083 & $2.0668(31)$ & $3.250(83)$ & $2.47(46)$ & $2.4(1.3)$ & - & $0.50(3)$ & $0.81(2)$ \\
\hline & 0.091 & $1.8835(28)$ & $2.746(70)$ & $2.28(43)$ & $3.3(1.8)$ & - & $0.48(3)$ & $0.83(2)$ \\
\hline \multirow[t]{2}{*}{ FL $(c$-like $)$} & 0.121 & $1.1533(8)$ & $1.308(9)$ & $1.20(4)$ & $12.9(4.5)$ & $1.4(2)$ & $1.38(5)$ & $0.23(2)$ \\
\hline & 0.127 & $0.9736(6)$ & $1.048(9)$ & $0.97(5)$ & $16.9(7.2)$ & $1.1(1)$ & $0.83(4)$ & $0.53(3)$ \\
\hline \multicolumn{9}{|l|}{ (b) Vector } \\
\hline \multirow[t]{4}{*}{ OK ( $b$-like) } & 0.039 & $2.2330(31)$ & $3.988(87)$ & $2.69(30)$ & $-4.5(1.0)$ & - & $1.36(4)$ & $0.23(2)$ \\
\hline & 0.040 & $2.1475(28)$ & $3.653(74)$ & $2.60(28)$ & $-4.1(1.1)$ & - & $1.23(4)$ & $0.29(2)$ \\
\hline & 0.041 & $2.0589(25)$ & $3.325(61)$ & $2.53(27)$ & $-3.7(1.2)$ & - & $1.20(4)$ & $0.31(2)$ \\
\hline & 0.042 & $1.9662(23)$ & $3.016(50)$ & $2.47(27)$ & $-3.1(1.3)$ & - & $1.18(4)$ & $0.31(2)$ \\
\hline \multirow[t]{4}{*}{ OK (c-like) } & 0.0468 & $1.4324(14)$ & $1.733(18)$ & $1.74(14)$ & $2.9(3.6)$ & - & $1.04(4)$ & $0.39(2)$ \\
\hline & 0.048 & $1.2594(13)$ & $1.443(15)$ & $1.48(10)$ & $4.5(5.7)$ & - & $0.97(4)$ & $0.45(2)$ \\
\hline & 0.049 & $1.0921(12)$ & $1.205(13)$ & $1.27(9)$ & $4.2(9.6)$ & - & $0.78(3)$ & $0.59(3)$ \\
\hline & 0.050 & $0.8920(14)$ & $0.969(15)$ & $0.98(7)$ & $-8.8(21.7)$ & - & $0.35(2)$ & $0.91(1)$ \\
\hline \multirow[t]{2}{*}{ FL (b-like) } & 0.083 & $2.0906(53)$ & $3.406(132)$ & $3.18(2.29)$ & $0.6(1.9)$ & - & $0.27(2)$ & $0.95(1)$ \\
\hline & 0.091 & $1.9157(37)$ & $2.890(74)$ & $2.68(91)$ & $1.0(2.9)$ & - & $0.54(3)$ & $0.78(2)$ \\
\hline \multirow[t]{2}{*}{ FL (c-like) } & 0.121 & $1.2287(15)$ & $1.438(19)$ & $1.43(10)$ & $10.7(5.5)$ & - & $0.58(3)$ & $0.75(2)$ \\
\hline & 0.127 & $1.0693(16)$ & $1.206(17)$ & $1.24(8)$ & $12.7(9.2)$ & - & $0.67(3)$ & $0.67(2)$ \\
\hline
\end{tabular}

$w_{4}=0$ at the tree level, so the two expressions in square brackets are of order $\alpha_{s}$. Because we use a tadpole-improved action, these one-loop errors are expected to be small.

We calculate the inconsistency $I$ from the pseudoscalar and vector masses presented in Sect. 4. The derivation of Eq. (40) does not include spin-dependent effects, which should contribute to $I$ for both pseudoscalar and vector channels. To separate spin-dependent effects, which are the subject of Sect. 6, from spin-independent ones, we form the spin-averaged mass,.

$\bar{M}=\frac{1}{4}\left(M+3 M^{*}\right)$,

writing $M\left(M^{*}\right)$ for pseudoscalar (vector) masses. We calculate the quantity $I$ with such spin-averaged rest and kinetic masses and plot the result in Fig. 4a. For orientation, we also show the physical $B_{s}$ and $D_{s}$ masses (for this ensemble) with vertical bands. We find that $I$ is close to 0 for the OK action even in the $b$-like region, whereas the Fermilab action leads to a very large deviation $I \approx-0.6$ from the continuum value, $I=0$, as in Ref. [24]. This outcome provides good numerical evidence that the improvement of spin-independent effects of the $\mathrm{OK}$ action is realized in practice. The small $I$ remaining stems from still higher-dimension kinetic operators of $\mathrm{O}\left(\lambda^{4}\right)$ in HQET, or $\mathrm{O}\left(v^{7}\right)$ in NRQCD, power counting, which are not addressed by the OK action. For completeness, Fig. 4b, c show $I$ for the pseudoscalar and vector channels, demonstrating that spin-dependent effects do not alter the conclusions.

Let us end this section with the "tuning wrinkle" mentioned in the introduction. By definition, Eq. (37), $M_{2}$ contains a binding energy, which, via $m_{4}$ in Eq. (40), is sensitive to the higher-dimension discretization errors addressed by the OK action. With the Fermilab action (as sometimes imple- 
Table 5 Dispersion fit results for quarkonium. The energies $E$ are from the correlator fits to the function in Eq. (30). The first column indicates the lattice action and the (approximate) heavy-quark flavor. The second column is the hopping parameter $\kappa$. The following columns are the rest mass $M_{1}$, the kinetic mass $M_{2}$, the generalized masses $M_{4}$ and $M_{6}$, and the coefficient of an $O(3)$ symmetry breaking terms $W_{4}$ and $W_{6}^{\prime}$. The last two columns are the $\chi^{2}$ divided by the degrees of freedom (dof) $N_{\text {data }}-N_{\text {param }}, p$-value. All ten momenta data points are included in the dispersion relation fit; these spectra are obtained from the correlator fits to Eq. (25), setting $R_{i}^{p}=0$, which include all correlations; $R_{1}=0$ for bottomonium fits. Fits to the dispersion relation include all correlations among different momenta. Errors are all from a singleelimination jackknife

\begin{tabular}{|c|c|c|c|c|c|c|c|c|c|}
\hline Action $(Q)$ & $\kappa$ & $M_{1}$ & $M_{2}$ & $M_{4}$ & $W_{4} \times 10^{2}$ & $M_{6}$ & $W_{6}^{\prime} \times 10^{4}$ & $\chi^{2} / \mathrm{dof}$ & $p$ \\
\hline \multicolumn{10}{|c|}{ (a) Pseudoscalar } \\
\hline \multirow[t]{4}{*}{ OK (b-like) } & 0.039 & $3.5200(4)$ & $6.637(19)$ & $4.58(8)$ & $-0.91(3)$ & - & - & $0.51(3)$ & $0.80(2)$ \\
\hline & 0.040 & $3.3616(4)$ & $6.120(17)$ & $4.38(8)$ & $-0.97(3)$ & - & - & $0.48(3)$ & $0.82(2)$ \\
\hline & 0.041 & $3.1968(4)$ & $5.601(16)$ & $4.16(8)$ & $-0.99(4)$ & - & - & $0.95(4)$ & $0.46(3)$ \\
\hline & 0.042 & $3.0242(4)$ & $5.083(14)$ & $3.90(7)$ & $-0.97(5)$ & - & - & $1.32(4)$ & $0.25(2)$ \\
\hline \multirow[t]{4}{*}{ OK (c-like) } & 0.0468 & $2.0169(5)$ & $2.647(11)$ & $2.41(9)$ & $0.7(4)$ & - & - & $0.59(3)$ & $0.74(2)$ \\
\hline & 0.048 & $1.6819(5)$ & $2.063(8)$ & $1.97(6)$ & $2.1(7)$ & - & - & $0.82(3)$ & $0.55(3)$ \\
\hline & 0.049 & $1.3516(5)$ & $1.574(5)$ & $1.54(3)$ & $3.6(1.5)$ & - & - & $1.20(4)$ & $0.30(2)$ \\
\hline & 0.050 & $0.9419(5)$ & $1.056(4)$ & $1.08(2)$ & $-0.6(4.1)$ & - & - & $1.36(4)$ & $0.23(2)$ \\
\hline \multirow[t]{2}{*}{ FL (b-like) } & 0.083 & $3.1765(3)$ & $9.392(23)$ & $3.69(2)$ & $1.84(2)$ & $4.1(1)$ & $5.6(3)$ & $0.77(3)$ & $0.55(2)$ \\
\hline & 0.091 & $2.8764(3)$ & $6.894(18)$ & $3.19(2)$ & $2.45(4)$ & $4.0(3)$ & $9.2(6)$ & $0.75(4)$ & $0.56(3)$ \\
\hline \multirow[t]{2}{*}{ FL $(c$-like $)$} & 0.121 & $1.6216(5)$ & $2.128(9)$ & $1.69(3)$ & $8.5(7)$ & $1.8(1)$ & - & $1.39(5)$ & $0.23(2)$ \\
\hline & 0.127 & $1.3087(5)$ & $1.561(6)$ & $1.35(2)$ & $12.0(1.5)$ & $1.4(1)$ & - & $0.83(4)$ & $0.53(3)$ \\
\hline \multicolumn{10}{|l|}{ (b) Vector } \\
\hline \multirow[t]{4}{*}{ OK (b-like) } & 0.039 & $3.5393(4)$ & $6.626(20)$ & $4.69(9)$ & $-0.99(3)$ & - & - & $0.64(3)$ & $0.70(2)$ \\
\hline & 0.040 & $3.3827(4)$ & $6.116(19)$ & $4.48(10)$ & $-1.04(4)$ & - & - & $0.69(3)$ & $0.66(2)$ \\
\hline & 0.041 & $3.2201(4)$ & $5.606(17)$ & $4.25(10)$ & $-1.05(5)$ & - & - & $1.03(4)$ & $0.40(2)$ \\
\hline & 0.042 & $3.0500(4)$ & $5.096(16)$ & $3.98(9)$ & $-1.00(6)$ & - & - & $1.36(4)$ & $0.23(2)$ \\
\hline \multirow[t]{4}{*}{ OK (c-like) } & 0.0468 & $2.0657(7)$ & $2.686(14)$ & $2.55(12)$ & $1.1(5)$ & - & - & $0.43(2)$ & $0.86(2)$ \\
\hline & 0.048 & $1.7425(7)$ & $2.112(10)$ & $2.08(9)$ & $2.8(9)$ & - & - & $0.49(3)$ & $0.81(2)$ \\
\hline & 0.049 & $1.4280(7)$ & $1.640(8)$ & $1.63(5)$ & $4.5(1.7)$ & - & - & $0.66(3)$ & $0.68(2)$ \\
\hline & 0.050 & $1.0497(9)$ & $1.154(7)$ & $1.15(3)$ & $-1.7(5.9)$ & - & - & $0.76(3)$ & $0.60(3)$ \\
\hline \multirow[t]{2}{*}{ FL (b-like) } & 0.083 & $3.1939(3)$ & $9.589(25)$ & $3.70(2)$ & $1.82(2)$ & $4.0(1)$ & $5.4(3)$ & $1.53(6)$ & $0.19(2)$ \\
\hline & 0.091 & $2.8987(4)$ & $7.035(20)$ & $3.21(2)$ & $2.43(4)$ & $4.0(3)$ & $9.0(5)$ & $0.44(3)$ & $0.78(2)$ \\
\hline \multirow[t]{2}{*}{ FL (c-like) } & 0.121 & $1.6774(8)$ & $2.235(13)$ & $1.75(5)$ & $7.6(9)$ & $1.9(2)$ & - & $1.05(4)$ & $0.39(2)$ \\
\hline & 0.127 & $1.3808(9)$ & $1.680(11)$ & $1.43(4)$ & $10.0(2.0)$ & $1.6(1)$ & - & $0.50(3)$ & $0.78(2)$ \\
\hline
\end{tabular}

mented [27,28]), a hadron-mass-based tuning of $\zeta$ transmits these errors from $M_{2}$ to $M_{1}$. This nuance has gone unappreciated; see, for example, Ref. [43].

\section{Hyperfine splittings}

The hyperfine splitting is the difference in the masses of the vector and pseudoscalar mesons:

$$
\begin{aligned}
& \Delta_{1}=M_{1}^{*}-M_{1}, \\
& \Delta_{2}=M_{2}^{*}-M_{2} .
\end{aligned}
$$

From Eq. (39), one has

$$
\Delta_{2}-\Delta_{1}=\delta B^{*}-\delta B .
$$

Spin-independent contributions cancel in this binding-energy difference, so the hyperfine difference $\Delta_{2}-\Delta_{1}$ diagnoses the improvement of the spin-dependent $c_{3}$ and $c_{5}$ terms of $\mathrm{O}\left(\lambda^{3}\right)$ in HQET power counting, or $\mathrm{O}\left(v^{6}\right)$ in NRQCD.

As one can see in Fig. 5a, the OK action shows clear improvement for quarkonium. The data points from the $\mathrm{OK}$ action lie much closer to the continuum value $\Delta_{2}=\Delta_{1}$ (the red line) for all simulated values of $\kappa_{\mathrm{OK}}$; in the charmonium region, they remain consistent with the continuum line within the error. The heavy-light results in Fig. 5b also show clear improvement in the region near the $D_{s}$ mass. The results with the $\mathrm{OK}$ action remain consistent with the continuum limit throughout the $B_{S}$ mass region, but the improvement is not yet statistically significant. Higher statistics would resolve the issue. All in all, the hyperfine splittings show the improve- 


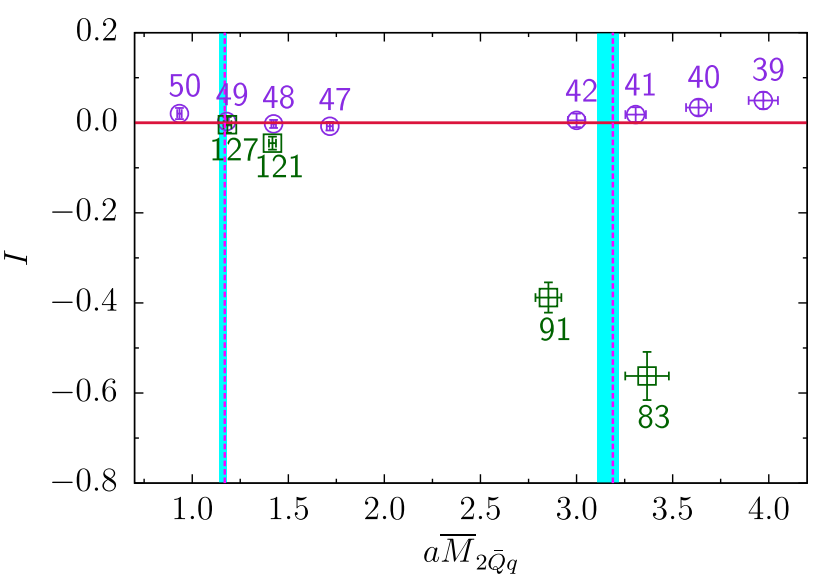

(a) Spin average

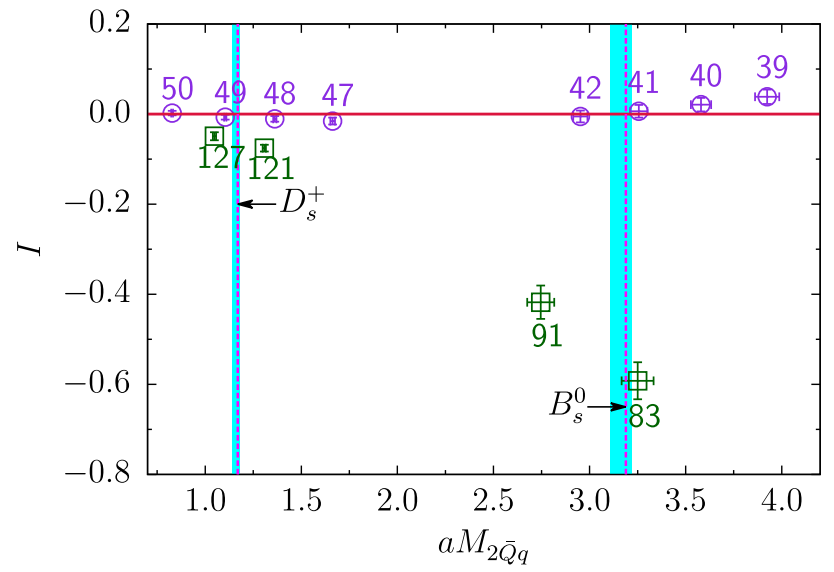

(b) Pseudoscalar

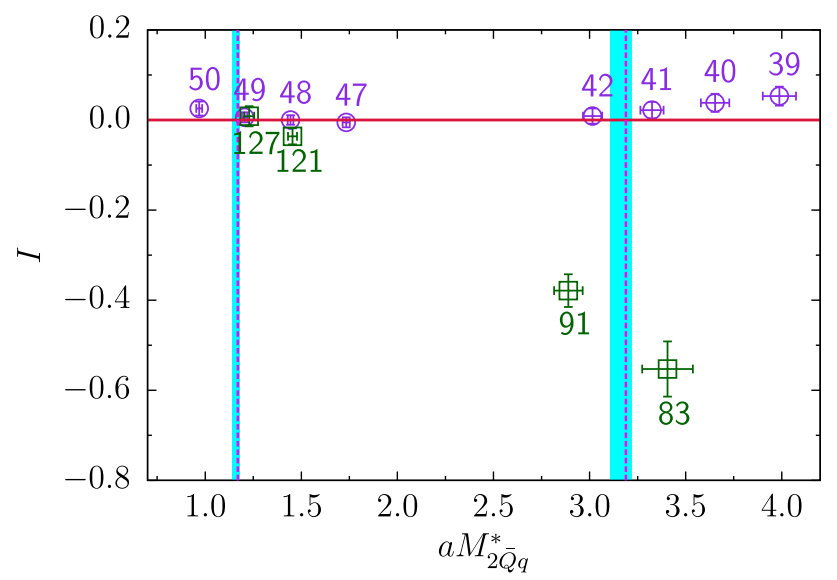

(c) Vector

Fig. 4 Inconsistency $I$ for a the spin-averaged mass $\bar{M}=\frac{1}{4}(M+$ $\left.3 M^{*}\right)$, b pseudoscalar-meson mass, and $\mathbf{c}$ vector-meson mass. Data labels denote the value of $\kappa \times 10^{3}$. The purple circles (green squares) represents the OK (Fermilab) action. The errors are from the jackknife. Vertical lines with bands represent the physical masses from the PDG [42] with experimental and (asymmetric) lattice-spacing errors added in quadrature. For the OK action $I$ almost vanishes (cf. horizontal red line), but for the Fermilab action it does not

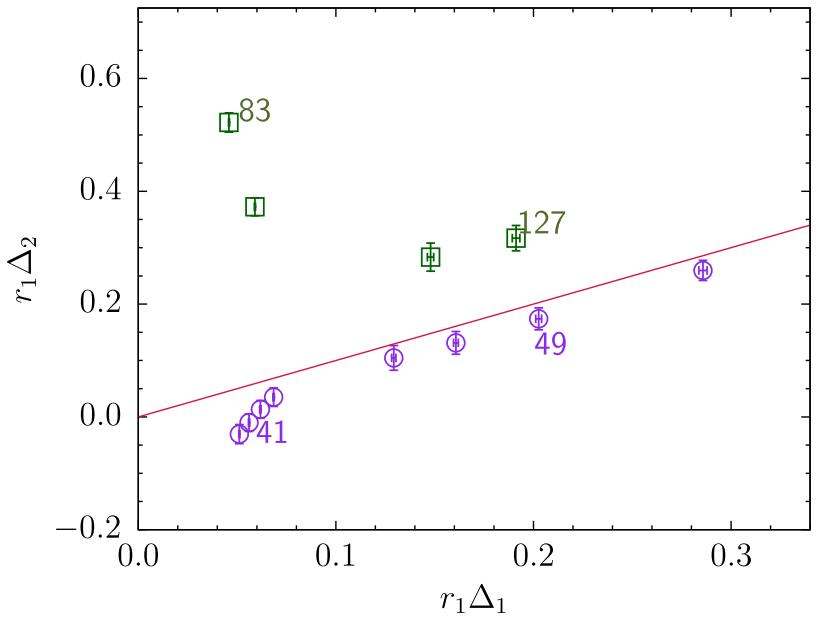

(a) Quarkonium

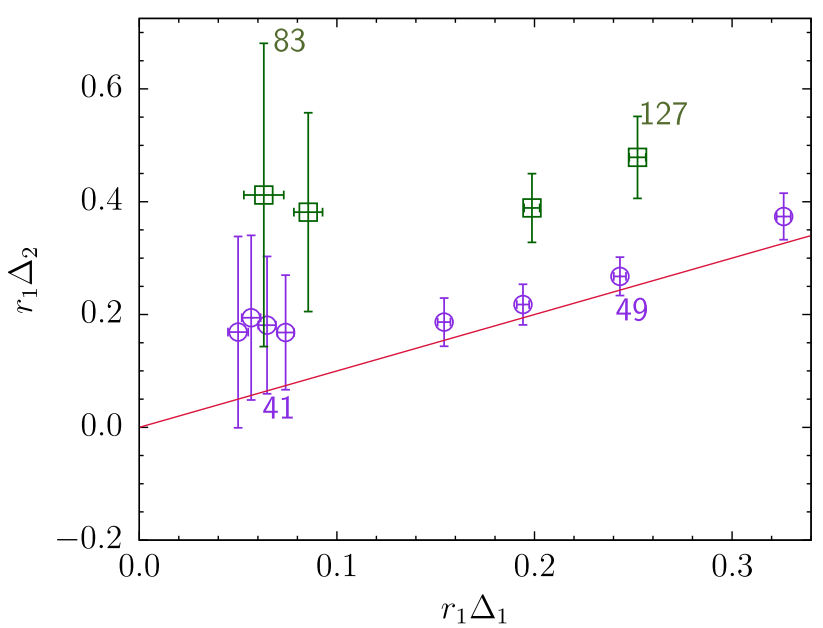

(b) Heavy-light meson

Fig. 5 Hyperfine splitting $\Delta_{2}$ obtained from the kinetic masses vs. $\Delta_{1}$, obtained from the rest masses. The square (green) represents the Fermilab action data, and the circle (purple) represents the OK action data. The labels are $\kappa \times 10^{3}$, corresponding to kinetic masses close to the physical $B_{s}(83,41)$ and $D_{s}(49,127)$ masses, as shown in Fig. 4. The continuum limit is represented by the line (red) $\Delta_{2}=\Delta_{1}$. Errors are from the jackknife

ment from the higher-dimension chromomagnetic interactions - those with couplings $c_{3}$ and $c_{5}$.

For both quarkonia and heavy-light mesons, the hyperfine splitting of the kinetic mass, $\Delta_{2}$, has a larger error than that of the rest mass, $\Delta_{1}$, because the kinetic mass requires correlators with $\boldsymbol{p} \neq \mathbf{0}$, which are noisier than those with $\boldsymbol{p}=\mathbf{0}$. As the rest mass $M_{1}$ and the kinetic mass $M_{2}$ are determined with smaller error with the OK action than the Fermilab action (see Sect. 4), the statistical errors shown for the hyperfine splittings $\Delta_{1}$ and $\Delta_{2}$ in Fig. 5 are smaller with the OK action, especially in the case of $\Delta_{2}$. 


\section{Conclusions}

Our tests of the Fermilab improvement program are based on the two-point correlators for mesons generated with the Fermilab [4] and OK [19] actions. Looking ahead to phenomenological applications [22,23,44], we have chosen four hopping parameters for the OK action in each of the $b$ - and $c$-quark mass regions. For the Fermilab action, we have simulated two $b$-like and two $c$-like hopping parameters. Although the lattice data span the physically interesting regions, the central aim of this paper is to test the improvement theory of Refs. [4,19] against simulation data, independent of the phenomenological interpretation of the action's parameters.

We focus on tests of both the spin-independent and the spin-dependent terms in the OK action. The results for the (spin-averaged) quantity $I$, known as the inconsistency [24], show that the OK action succeeds in improving the effects that generate the kinetic-mass binding energy. The hyperfine splitting shows that the OK action significantly improves the higher-dimension spin-dependent, chromomagnetic effects, at least in quarkonium. For the heavy-light system, the data show a clear improvement for smaller $c$-like masses, but in the $b$-like region, large statistical errors prevent us from reaching firm conclusions.

This study has yielded some noteworthy byproducts. As $a m_{0}$ is reduced, here with fixed $a \approx 0.12 \mathrm{fm}$, the meson masses $M_{1}, M_{2}$, and $M_{4}$ approach each other, verifying expectations for the Fermilab and $\mathrm{OK}$ actions. The difference between $M_{4}$ and $M_{2}$, for $a m_{2} \ll 1$, is not much smaller for the OK action than the Fermilab action. By analogy with the inconsistency $I$ [24,25], this feature is probably explained by the associated binding energy $M_{4}-m_{4}$, which stems from higher-dimension effects not improved by the OK action. Finally, in the $b$-like region, $a m_{2} \sim 3$, the OK action produces statistically more precise results than the Fermilab action for the heavy-light correlator energies and, hence, the masses $M_{2}$ and $M_{4}$.

As an application of the OK action, a calculation of the form factors for the $B \rightarrow D^{(*)} \ell v$ semileptonic decays is under way, with the aim of determining the CKM matrix element $\left|V_{c b}\right|$. To achieve the desired sub-percent precision on the relevant form factors, it will be necessary to derive the analog of the OK action for currents [22,23], and to calculate the renormalization. Meanwhile some of us are extending the present work to a full-fledged tuning run [44].

Acknowledgements J.A.B. is supported by the Basic Science Research Program of the National Research Foundation of Korea (NRF) funded by the Ministry of Education (No. 2015024974). This project is supported in part by the U.S. Department of Energy under Grant No. DE-FC02-12ER-41879 (C.D.) and the U.S. National Science Foundation under Grant No. PHY14-14614 (C.D.). A.S.K. is supported in part by the German Excellence Initiative and the European Union Seventh Framework Programme under Grant Agreement No. 291763 as well as the European Union's Marie Curie COFUND program. This manuscript has been co-authored by an employee of Fermi Research Alliance, LLC, under Contract No. DE-AC02-07CH1 1359 with the U.S. Department of Energy, Office of Science, Office of High Energy Physics. The United States Government retains and the publisher, by accepting the article for publication, acknowledges that the United States Government retains a non-exclusive, paid-up, irrevocable, world-wide license to publish or reproduce the published form of this manuscript, or allow others to do so, for United States Government purposes. The research of W.L. is supported by the Creative Research Initiatives Program (No. 20160004939) of the NRF grant funded by the Korean government (MEST). W.L. would like to acknowledge the support from KISTI supercomputing center through the strategic support program for the supercomputing application research (No. KSC-2014-G3-003). Further computations were carried out on the DAVID GPU clusters at Seoul National University.

Open Access This article is distributed under the terms of the Creative Commons Attribution 4.0 International License (http://creativecomm ons.org/licenses/by/4.0/), which permits unrestricted use, distribution, and reproduction in any medium, provided you give appropriate credit to the original author(s) and the source, provide a link to the Creative Commons license, and indicate if changes were made. Funded by SCOAP ${ }^{3}$.

\section{References}

1. S. Aoki et al., Eur. Phys. J. C 77(2), 112 (2017). https://doi.org/10. 1140/epjc/s10052-016-4509-7

2. A. Bazavov et al., Phys. Rev. D 93(11), 113016 (2016). https://doi. org/10.1103/PhysRevD.93.113016

3. A.S. Kronfeld, Nucl. Phys. B Proc. Suppl. 129, 46 (2004). https:// doi.org/10.1016/S0920-5632(03)02506-4

4. A.X. El-Khadra, A.S. Kronfeld, P.B. Mackenzie, Phys. Rev. D 55, 3933 (1997). https://doi.org/10.1103/PhysRevD.55.3933

5. G.P. Lepage, B.A. Thacker, Nucl. Phys. B Proc. Suppl. 4, 199 (1988). https://doi.org/10.1016/0920-5632(88)90102-8

6. B.A. Thacker, G.P. Lepage, Phys. Rev. D 43, 196 (1991). https:// doi.org/10.1103/PhysRevD.43.196

7. C.T.H. Davies, B.A. Thacker, Phys. Rev. D 45, 915 (1992). https:// doi.org/10.1103/PhysRevD.45.915

8. G.P. Lepage, L. Magnea, C. Nakhleh, U. Magnea, K. Hornbostel, Phys. Rev. D 46, 4052 (1992). https://doi.org/10.1103/PhysRevD. 46.4052

9. G.T. Bodwin, E. Braaten, G.P. Lepage, Phys. Rev. D 51, 1125 (1995). https://doi.org/10.1103/PhysRevD.51.1125. https:// doi.org/10.1103/PhysRevD.55.5853

10. E. Eichten, Nucl. Phys. B Proc. Suppl. 4, 170 (1988). https://doi. org/10.1016/0920-5632(88)90097-7

11. E. Eichten, B.R. Hill, Phys. Lett. B 234, 511 (1990). https://doi. org/10.1016/0370-2693(90)92049-O

12. E. Eichten, B.R. Hill, Phys. Lett. B 240, 193 (1990). https://doi. org/10.1016/0370-2693(90)90432-6

13. B. Grinstein, Nucl. Phys. B 339, 253 (1990). https://doi.org/10. 1016/0550-3213(90)90349-I

14. A.S. Kronfeld, Phys. Rev. D 62, 014505 (2000). https://doi.org/10. 1103/PhysRevD.62.014505

15. J. Harada, S. Hashimoto, K.I. Ishikawa, A.S. Kronfeld, T. Onogi, N. Yamada, Phys. Rev. D 65, 094513 (2002). https://doi.org/10.1103/ PhysRevD.65.094513 [Erratum: Phys. Rev. D 71, 019903 (2005). https://doi.org/10.1103/PhysRevD.71.019903]

16. J. Harada, S. Hashimoto, A.S. Kronfeld, T. Onogi, Phys. Rev. D 65, 094514 (2002). https://doi.org/10.1103/PhysRevD.65.094514 
17. T. Burch, C. DeTar, M. Di Pierro, A.X. El-Khadra, E.D. Freeland, S. Gottlieb, A.S. Kronfeld, L. Levkova, P.B. Mackenzie, J.N. Simone, Phys. Rev. D 81, 034508 (2010). https://doi.org/10.1103/ PhysRevD.81.034508

18. K. Symanzik, Nucl. Phys. B 226, 187 (1983). https://doi.org/10. 1016/0550-3213(83)90468-6

19. M.B. Oktay, A.S. Kronfeld, Phys. Rev. D 78, 014504 (2008). https://doi.org/10.1103/PhysRevD.78.014504

20. K.G. Wilson, in New Phenomena in Subnuclear Physics, ed. by A. Zichichi (Plenum, New York, 1977), pp. 69-142

21. B. Sheikholeslami, R. Wohlert, Nucl. Phys. B 259, 572 (1985). https://doi.org/10.1016/0550-3213(85)90002-1

22. J.A. Bailey, J. Leem, W. Lee, Y.C. Jang, PoS LATTICE2016, 285 (2016)

23. J.A. Bailey, Y.C. Jang, W. Lee, J. Leem, PoS LATTICE2014, 389 (2015)

24. S. Collins, R.G. Edwards, U.M. Heller, J.H. Sloan, Nucl. Phys. B Proc. Suppl. 47, 455 (1996). https://doi.org/10.1016/ 0920-5632(96)84680-9

25. A.S. Kronfeld, Nucl. Phys. B Proc. Suppl. 53, 401 (1997). https:// doi.org/10.1016/S0920-5632(96)00671-8

26. C. Bernard et al., Phys. Rev. D 83, 034503 (2011). https://doi.org/ 10.1103/PhysRevD.83.034503

27. S. Aoki, Y. Kuramashi, S.I. Tominaga, Prog. Theor. Phys. 109, 383 (2003). https://doi.org/10.1143/PTP.109.383

28. N.H. Christ, M. Li, H.W. Lin, Phys. Rev. D 76, 074505 (2007). https://doi.org/10.1103/PhysRevD.76.074505

29. A. Bazavov et al., Rev. Mod. Phys. 82, 1349 (2010). https://doi. org/10.1103/RevModPhys.82.1349

30. Y.C. Jang, J.A. Bailey, W. Lee, C. DeTar, M.B. Oktay, A.S. Kronfeld, PoS LATTICE2013, 030 (2014)
31. C. DeTar, A.S. Kronfeld, M.B. Oktay, PoS LATTICE2010, 234 (2010)

32. J.A. Bailey, Y.C. Jang, W. Lee, C. DeTar, A.S. Kronfeld, M.B. Oktay, PoS LATTICE2014, 097 (2014)

33. J.A. Bailey, Y.C. Jang, W. Lee, C. DeTar, A.S. Kronfeld, M.B. Oktay, PoS LATTICE2015, 099 (2016)

34. G.P. Lepage, P.B. Mackenzie, Phys. Rev. D 48, 2250 (1993). https:// doi.org/10.1103/PhysRevD.48.2250

35. http://www.usqcd.org/usqcd-software (retrieved August 2017)

36. S. Naik, Nucl. Phys. B 316, 238 (1989). https://doi.org/10.1016/ 0550-3213(89)90394-5

37. T. Blum, C.E. Detar, S.A. Gottlieb, K. Rummukainen, U.M. Heller, J.E. Hetrick, D. Toussaint, R.L. Sugar, M. Wingate, Phys. Rev. D 55, 1133 (1997). https://doi.org/10.1103/PhysRevD.55.1133

38. K. Orginos, D. Toussaint, Phys. Rev. D 59, 014501 (1999). https:// doi.org/10.1103/PhysRevD.59.014501

39. G.P. Lepage, Phys. Rev. D 59, 074502 (1999). https://doi.org/10. 1103/PhysRevD.59.074502

40. K. Orginos, D. Toussaint, R.L. Sugar, Phys. Rev. D 60, 054503 (1999). https://doi.org/10.1103/PhysRevD.60.054503

41. M. Wingate, J. Shigemitsu, C.T.H. Davies, G.P. Lepage, H.D. Trottier, Phys. Rev. D 67, 054505 (2003). https://doi.org/10.1103/ PhysRevD.67.054505

42. C. Patrignani et al., Chin. Phys. C 40(10), 100001 (2016). https:// doi.org/10.1088/1674-1137/40/10/100001

43. Y. Aoki et al., Phys. Rev. D 86, 116003 (2012). https://doi.org/10. 1103/PhysRevD.86.116003

44. H. Jeong, W. Lee, J. Leem, S. Park, T. Bhattacharya, R. Gupta, Y.C. Jang, PoS LATTICE2016, 380 (2016) 